\title{
Graphene Quantum Dots: Physico-Chemical Characterization and In Vitro Biological Effects ${ }^{\dagger}$
}

\author{
Miruna-Silvia Stan ${ }^{1,2,3, *}$, Beatrice G. Sbarcea ${ }^{4, *}$, Bogdan Trica ${ }^{5}$ and Anca Dinischiotu 1 \\ 1 Department of Biochemistry and Molecular Biology, Faculty of Biology, University of Bucharest, \\ 91-95 Splaiul Independentei, 050095 Bucharest, Romania; anca.dinischiotu@bio.unibuc.ro \\ 2 Department of Science and Engineering of Oxide Materials and Nanomaterials, \\ Faculty of Applied Chemistry and Materials Science, University Politehnica of Bucharest, \\ 1-7 Polizu Street, 011061 Bucharest, Romania \\ 3 Research Institute of the University of Bucharest-ICUB, University of Bucharest, \\ 91-95 Splaiul Independentei, 050095 Bucharest, Romania \\ 4 Advanced Materials Department, National Institute for R\&D in Electrical Engineering (ICPE-CA), \\ 030138 Bucharest, Romania \\ 5 The National Institute for Research \& Development in Chemistry and Petrochemistry (INCDCP-ICECHIM), \\ 030138 Bucharest, Romania; trica.bogdan@gmail.com \\ * Correspondence: miruna.stan@bio.unibuc.ro (M.-S.S.); gabi_bea@yahoo.com (B.G.S.) \\ + Presented at the 2nd International Online-Conference on Nanomaterials, 15-30 November 2020; Available \\ online: https://iocn2020.sciforum.net/.
}

Citation: Stan, M.-S.; Sbarcea, B.G.; Trica, B.; Dinischiotu, A. Graphene Quantum Dots: Physico-Chemical Characterization and In Vitro Biological Effects. Mater. Proc. 2021, 4, 27. https://doi.org/10.3390/ IOCN2020-07911

Academic Editors: Ana María Díez-Pascual, Antonio Di Bartolomeo and Guanying Chen

Published: 11 November 2020

Publisher's Note: MDPI stays neutral with regard to jurisdictional claims in published maps and institutional affiliations.

Copyright: $@ 2020$ by the authors. Licensee MDPI, Basel, Switzerland. This article is an open access article distributed under the terms and conditions of the Creative Commons Attribution (CC BY) license (http://creativecommons.org/licenses/by/4.0/).

\begin{abstract}
Graphene quantum dots (GQDs) represent nanoscale structures with strong quantum and exceptional photoluminescence properties. These particles have promising applications in nanomedicine, specifically for diagnostics, cargo delivery, photothermal therapy and bioimaging. In this context, we aimed to characterize GQDs available on the market for further utilization for in vivo purposes. Transmission and scanning electron microscopy (TEM and SEM), and energy dispersive X-ray spectroscopy (EDX), were used to characterize the morphology and elemental composition of GQDs. In addition, the hydrodynamic size and the zeta potential were measured for these nanoparticles. Their biocompatibility was investigated on human fibroblast lung cells (MRC-5 cell line) after 24 and $72 \mathrm{~h}$ of incubation with concentrations up to $200 \mu \mathrm{g} / \mathrm{mL}$ of GQDs. TEM images showed graphene sheets with few wrinkle structures, the dots having uniform diameters in the range between 1.0 and $5.0 \mathrm{~nm}$. SEM examination revealed the three-dimensional structure with a sponge-like aspect and pores of various sizes. Their tendency to aggregate provided the formation of aggregates with sizes of hundreds of nanometers, as revealed by the hydrodynamic diameter of about $270 \mathrm{~nm}$. A negative zeta potential of $-16 \mathrm{mV}$ confirmed the anionic character of GQDs. Concentrations up to $50 \mu \mathrm{g} / \mathrm{mL}$ exhibited a low toxicity in lung cells, as revealed by MTT assay and fluorescent microscopy of actin cytoskeleton after both time intervals, confirming potential further testing on animals for clinical purposes. However, the high doses of GQDs induced cell death and must be avoided in future. Given the new experimental evidence obtained on GQDs, more knowledge has been achieved, which is very useful for prospective research to revolutionize the future of nanomedicine and biotechnology.
\end{abstract}

Keywords: graphene; quantum dots; biocompatibility

\section{Introduction}

Graphene quantum dots (GQDs) represent nanoscale structures with strong quantum and exceptional photoluminescence properties. These particles have promising applications in nanomedicine, specifically, for diagnostics, cargo delivery, photothermal therapy and bioimaging [1,2]. In this context, we aimed to characterize GQDs available on the market for further utilization for in vivo purposes. 


\section{Materials and Methods}

Transmission and scanning electron microscopy (TEM and SEM), and energy dispersive X-ray spectroscopy (EDX), were used to characterize the morphology and elemental composition of GQDs. In addition, the hydrodynamic size and the zeta potential were measured for these nanoparticles. Their biocompatibility was investigated on human fibroblast lung cells (MRC-5 cell line) after 24 and $72 \mathrm{~h}$ of incubation with concentrations of up to $200 \mu \mathrm{g} / \mathrm{mL}$ of GQDs.

\section{Results}

TEM images showed graphene sheets with few wrinkle structures, the dots having uniform diameters in the range between 1.0 and $5.0 \mathrm{~nm}$. SEM examination revealed the three-dimensional structure with a sponge-like aspect and pores of various sizes. Their tendency to aggregate provided the formation of aggregates with sizes of hundreds of nanometers, as revealed by the hydrodynamic diameter of about $270 \mathrm{~nm}$. A negative zeta potential of $-16 \mathrm{mV}$ confirmed the anionic character of GQDs.

Concentrations up to $50 \mu \mathrm{g} / \mathrm{mL}$ exhibited a low toxicity in lung cells, as revealed by the MTT assay, phase contrast images (Figure 1) and fluorescent microscopy of actin cytoskeleton (Figure 2) after both time intervals, confirming potential further testing on animals for clinical purposes. However, the high doses of GQDs induced cell death and must be avoided in future.
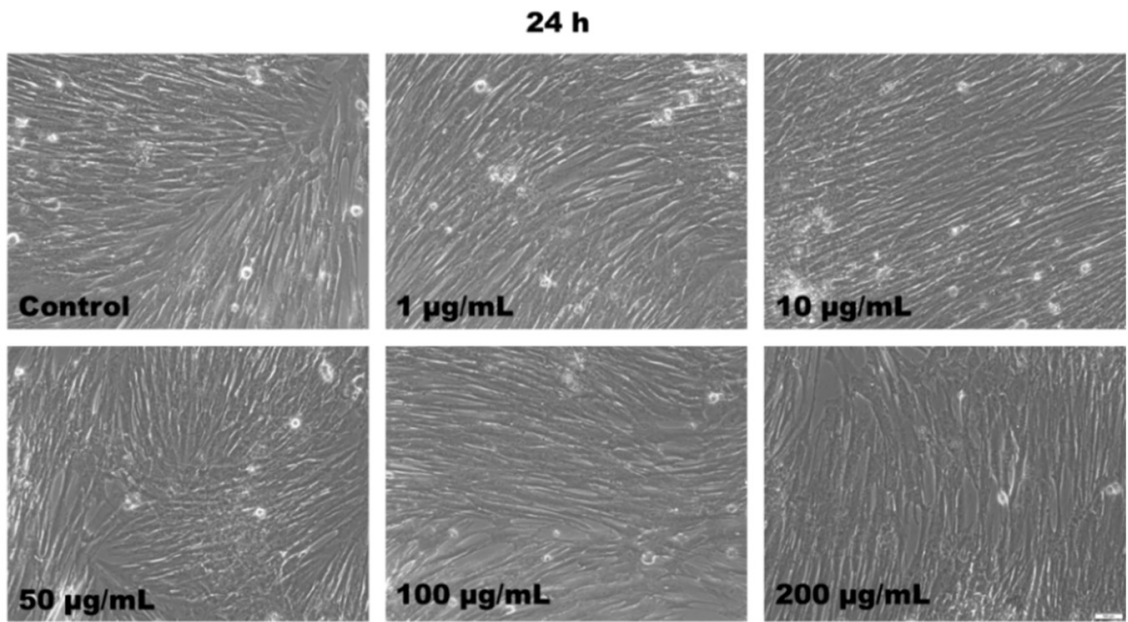

(a)

$72 \mathrm{~h}$
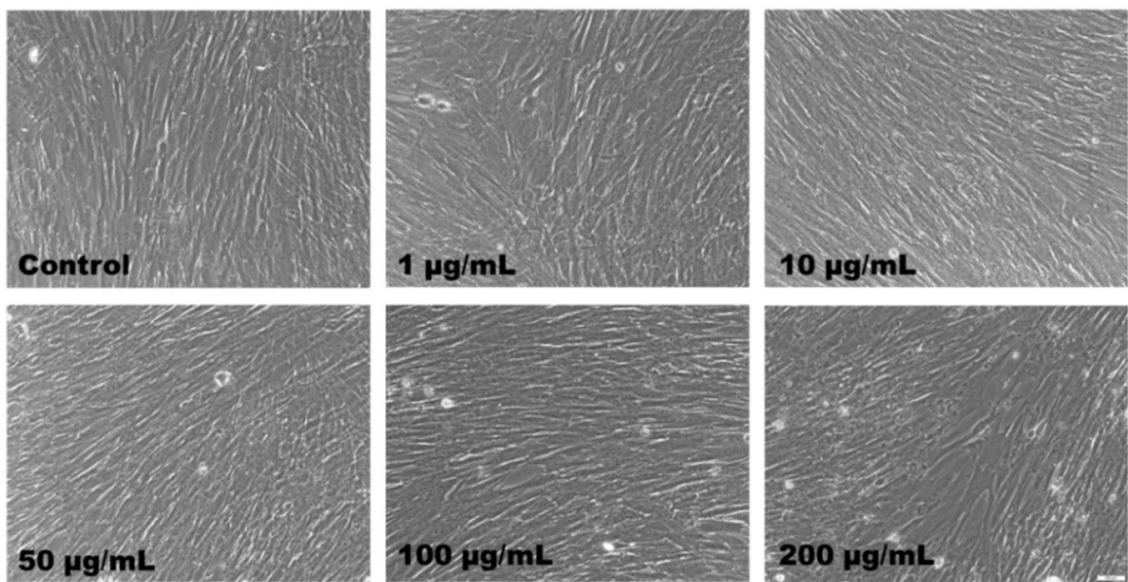

(b)

Figure 1. Phase contrast images of MRC-5 cells incubated with graphene quantum dots (GQDs) for 24 (a) and $72 \mathrm{~h}$ (b). 

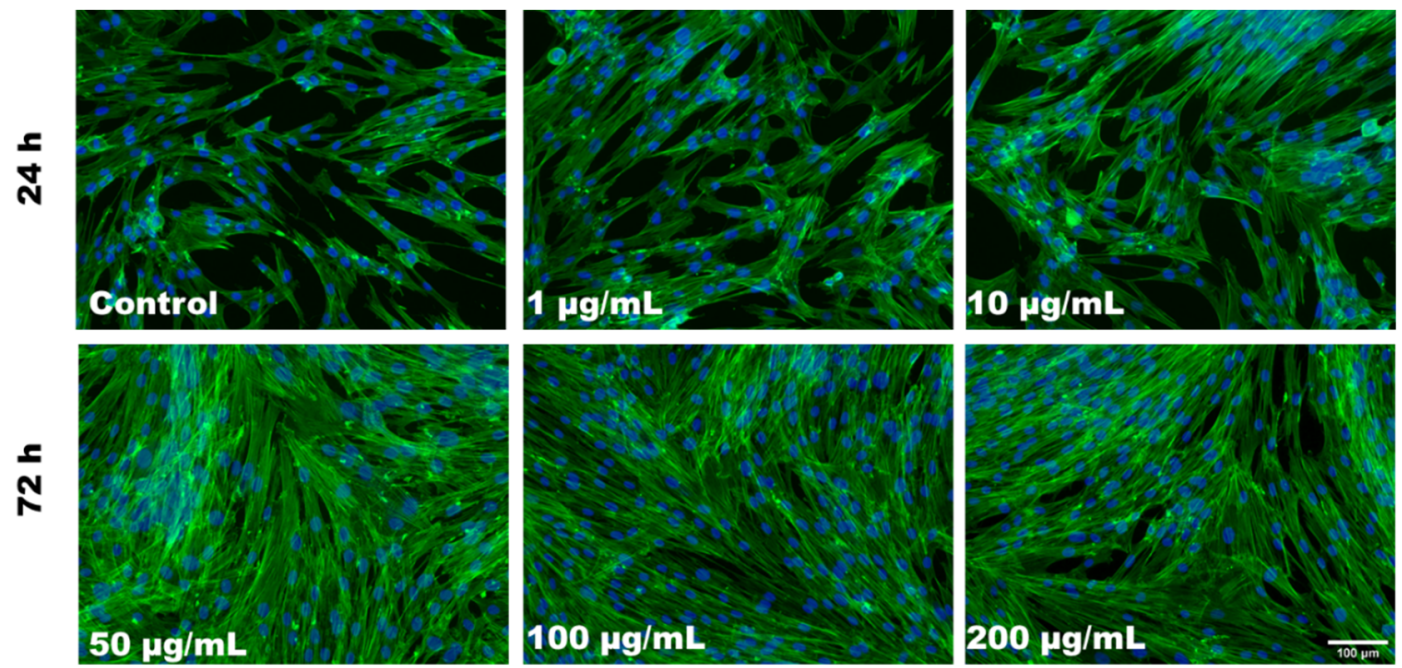

Figure 2. Fluorescence images of F-actin in MRC-5 cells incubated with GQDs for 24 and $72 \mathrm{~h}$.

\section{Conclusions}

Given the new experimental evidence obtained on GQDs, more knowledge has been achieved, which is very useful for prospective research to revolutionize the future of nanomedicine and biotechnology.

Supplementary Materials: The following are available online at https://www.mdpi.com/article/10.3390/IOCN2020-07911/s1.

Funding: M.S. Stan acknowledges the ICUB grant for young researchers (no. 20971/30.10.2020) and the support of the Operational Programme Human Capital of the Ministry of European Funds through the Financial Agreement 51668/09.07.2019, SMIS code 124705.

Institutional Review Board Statement: Not applicable.

Informed Consent Statement: Not applicable.

Data Availability Statement: The data is available on the request from the corresponding author.

Conflicts of Interest: The authors declare no conflicts of interest. The funders had no role in the design of the study; in the collection, analyses, or interpretation of data; in the writing of the manuscript, or in the decision to publish the results.

\section{References}

1. Zhao, C.; Song, X.; Liu, Y.; Fu, Y.; Ye, L.; Wang, N.; Wang, F.; Li, L.; Mohammadniaei, M.; Zhang, M.; et al. Synthesis of graphene quantum dots and their applications in drug delivery. J. Nanobiotechnol. 2020, 18, 142.

2. Jampilek, J.; Kralova, K. Advances in drug delivery nanosystems using graphene-based materials and carbon nanotubes. Materials 2021, 14, 1059. 